\title{
SLC25A12 and CMYA3 gene variants are not associated with autism in the IMGSAC multiplex family sample
}

\author{
Francesca Blasi ${ }^{1,2,5}$, Elena Bacchelli ${ }^{1,2,5}$, Simona Carone ${ }^{1,2}$, Claudio Toma ${ }^{1,2}$, \\ Anthony P Monaco ${ }^{3}$, Anthony J Bailey ${ }^{4}$, Elena Maestrini ${ }^{*, 1,2}$ and the International \\ Molecular Genetic Study of Autism Consortium (IMGSAC) ${ }^{6}$
}

\begin{abstract}
${ }^{1}$ Department of Biology, University of Bologna, Bologna, Italy; ${ }^{2}$ Medical Genetics Laboratory, Policlinico $S$ Orsola, Bologna, Italy; ${ }^{3}$ Wellcome Trust Centre for Human Genetics, University of Oxford, Oxford, UK; ${ }^{4}$ Section of Child and Adolescent Psychiatry, University Department of Psychiatry, Park Hospital for Children, Oxford, UK
\end{abstract}

Autism is a severe neurodevelopmental disorder with a complex genetic predisposition. Linkage findings from several genome scans suggest the presence of an autism susceptibility locus on chromosome 2q24-q33, making this region the focus of candidate gene and association studies. Recently, significant association with autism has been reported for single-nucleotide polymorphisms (SNPs) in the SLC25A12 and CMYA3 genes on chromosome $2 q$. We attempted to replicate these findings in the collection of families from the International Molecular Genetic Study of Autism Consortium (IMGSAC), using the transmission disequilibrium test and case-control comparison. Our study failed to reveal any significant association for the SNPs tested at either locus, suggesting that these variants are unlikely to play a major role in genetic susceptibility to autism in our sample.

European Journal of Human Genetics (2006) 14, 123-126. doi:10.1038/sj.ejhg.5201444; published online 5 October 2005

Keywords: autism; chromosome 2q; association; SLC25A12; CMYA3; SNPs

\section{Introduction}

Autism is a neurodevelopmental disorder characterized by a combination of qualitative impairments in communication and reciprocal social interaction, and by restricted and repetitive behaviours and interests; onset is in the first 3 years of life. Recent epidemiological studies have indicated a population prevalence for autism of about 10 in 10000 , although the prevalence of all forms of pervasive developmental disorders is around six times higher. There is

\footnotetext{
${ }^{*}$ Correspondence: Dr E Maestrini, Dipartimento di Biologia Evoluzionistica Sperimentale, Via Selmi 3, Bologna 40126, Italy.

Tel: + 390512094 178; Fax: + 390512094 286;

E-mail: maestrin@alma.unibo.it

${ }^{5}$ These authors contributed equally to this work

${ }^{6}$ http://www.well.ox.ac.uk/ maestrin/iat.html

Received 1 December 2004; revised 15 April 2005; accepted 19 April 2005; published online 5 October 2005
}

convincing evidence from twin and family studies for a complex genetic predisposition to autism, and statistical modelling suggests the involvement of perhaps 3 or 4 loci, although up to 15 loci may be implicated. ${ }^{1}$

Several groups have undertaken genome-wide scans for autism susceptibility loci using affected relative pairs (ARP), and a number of chromosomal regions with converging evidence of linkage have been identified, most notably on chromosomes $2 \mathrm{q}$ and $7 \mathrm{q}$. In the genome screen performed by the International Molecular Genetic Study of Autism Consortium (IMGSAC), the strongest evidence for linkage was in the 2q24-q33 region, with a multipoint maximum lod score (MLS) of 3.74 at marker D2S2188 in a sample of 152 affected sib-pairs (ASP). ${ }^{2}$ In an expanded sample of 219 ASP, the peak MLS was 2.54 near D2S2314 in 2q31. ${ }^{3}$ Additional support for the presence of an autism susceptibility locus on chromosome $2 \mathrm{q}$ is given by overlapping linkage findings in another three independent genome scans. ${ }^{4-6}$ 
The multiple linkage findings on chromosome 2 have made this region the focus of candidate gene and association studies. Recently, very promising results have been obtained for two genes: SLC25A12 and CMYA3. Ramoz et $\mathrm{al}^{7}$ reported a strong association of autism with two intronic single-nucleotide polymorphisms (SNPs) within SLC25A12, a gene encoding the mitochondrial aspartate/glutamate carrier. The two SNPs (rs2056202 and rs2292813) were identified through mutation screening of eight candidate genes mapping across the $2 \mathrm{q} 31$ region in affected individuals chosen from 38 families showing linkage to this interval. Association analysis in 411 autistic families using the transmission disequilibrium test (TDT) revealed significant excess transmission for the common allele of both SNPs and the corresponding haplotype $(\mathrm{G}-\mathrm{G})\left(P=3 \times 10^{-6}\right)$.

Evidence for the involvement in autism of CMYA3, a widely expressed gene of unknown function, has been obtained using an innovative high-throughput technology for large-scale variation scanning called variation scanning on tag arrays (VSTA). ${ }^{8}$ The authors screened the exons of all known genes mapping in a $20 \mathrm{Mb}$ region in $2 \mathrm{q} 24.3-\mathrm{q} 32.1$ for sequence variants, in a collection of 372 patients with autism and 404 controls. A nonsynonymous substitution in CMYA3 (rs3749004) was identified as their most interesting result $\left(P=3.13 \times 10^{-6}\right){ }^{8}$

The evidence for significant association between autism and SNPs within genes mapping in a region of linkage supports the hypothesis that SLC25A12 and CMYA3 influence genetic risk in autism. As with all complex disorders, independent replication is essential to confirm these results. Thus, we decided to follow-up these association results in the collection of families from the IMGSAC.

\section{Materials and methods Subjects}

The identification of families and assessment methods used by the IMGSAC has been previously described in detail. ${ }^{2}$ A total of 261 Caucasian multiplex families have been genotyped for this study, comprising 225 ASP families and 36 other ARP families. The total number of affected individuals screened is 531 .

The control sample consisted of 174 DNAs of a collection available from the European Collection of Cell Cultures (ECACC), derived from randomly selected Caucasian blood donors whose parents and grandparents were born in the UK or Ireland.

\section{SNPs typing}

Sample genotypes were determined using the multiplexed ligation detection reaction technique (LDR) as described previously. ${ }^{9}$ PCR products containing the SNPs were generated using the primers shown in Table 1. For LDR genotyping, three oligonucleotide probes were designed for each SNP: these comprise two labelled allele-specific probes and a phosphorylated unlabelled common probe (Table 1). If required, the length of probes was adjusted by adding polyA's or polyT's not complementary to the sequence (lower-case letters in Table 1). Before LDR amplification, $1 \mu \mathrm{l}$ of PCR products for each of the three SNPs were pooled in a final volume of $9 \mu \mathrm{l}$, and treated with $1 \mu \mathrm{l}$ of $1 \mathrm{mg} / \mathrm{ml}$ proteinase $\mathrm{K}$ in $10 \mathrm{~mm}$ EDTA at $37^{\circ} \mathrm{C}$ for $15 \mathrm{~min}, 55^{\circ} \mathrm{C}$ for $10 \mathrm{~min}$ and $90^{\circ} \mathrm{C}$ for $10 \mathrm{~min}$. LDR was performed in a final volume of $7 \mu \mathrm{l}$, containing $2.5 \mu \mathrm{l}$ of PCR pool, $0.05 \mu \mathrm{M}$ phosphorylated probes, $0.017 \mu \mathrm{M}$ allellespecific probes and 0.2 U Ampligase DNA ligase (CAMBIO) for each SNP. Reactions were incubated for $1 \mathrm{~min}$ at $95^{\circ} \mathrm{C}$ followed by 15 cycles of $15 \mathrm{~s}$ at $95^{\circ} \mathrm{C}$ and $4 \mathrm{~min}$ at $61^{\circ} \mathrm{C}$. Finally, $1 \mu \mathrm{l}$ of each LDR amplification product was run on an ABI prism 3730 sequencer (Applied Biosystems) and genotypes analysed using GENEMAPPER ${ }^{\mathrm{TM}}$ software (Applied Biosystems).

\section{Statistical analysis}

After checking genotypes for Mendelian consistency and Hardy-Weinberg equilibrium, multipoint lod scores were computed using ASPEX sib_phase under an additive model (no dominance variance). TDT analysis was performed using the sib_tdt program from ASPEX v2.3. ${ }^{10}$ This

Table 1 PCR and LDR oligonucleotides

\begin{tabular}{|c|c|c|c|}
\hline SNP ID & PCR primers & LDR probes & LDR size (bp) \\
\hline rs3749004 & $\begin{array}{l}\text { F: GATTTCCTCTTGAGCGTCCA } \\
\text { R: CCGACGAACAGAAAACCCTA }\end{array}$ & $\begin{array}{l}\text { A1: (FAM)-GGGTTGCTGCTGGACATGGGAC } \\
\text { A2: (NED)-GGGTTGCTGCTGGACATGGGAT } \\
\text { C: GGCCCCTTTGGATAACTTTGCAGT }\end{array}$ & 46 \\
\hline rs2292813 & $\begin{array}{l}\text { F: GAGTTGCAACAATGCTITTGTAGA } \\
\text { R: GGGCTGGAGCCATACAGAAT }\end{array}$ & $\begin{array}{l}\text { A1: (FAM)-ACTTGAAACCAGGACAAATGTGGTAAAGAC } \\
\text { A2: (NED)-ACTTGAAACCAGGACAAATGTGGTAAAGAT } \\
\text { C: AGCCACTGAGTCACAGGGGAGGAC }\end{array}$ & 54 \\
\hline rs2056202 & $\begin{array}{l}\text { F: GAAGACTGGACCAGCATCATT } \\
\text { R: TCACCAGTACTGAGGTTGAGAAA }\end{array}$ & $\begin{array}{l}\text { A1: (FAM)-ATAGGAGATCAACCTGGAATAAAATATAAAACGT } \\
\text { A2: (NED)-tatGGAGATCAACCTGGAATAAAATATAAAACGC } \\
\text { C: CATTGGCTGGTGAAGAAAGGGAAAG }\end{array}$ & 59 \\
\hline
\end{tabular}


program calculates probabilities for $\chi^{2}$ statistics by permuting parental alleles while fixing the IBD status of siblings within a family, thereby allowing the use of multiple siblings within a nuclear family. TDT analysis included all affected siblings from ASP families and a single randomly selected affected individual from each of the 36 ARP families. The extent of linkage disequilibrium (LD) between the intragenic markers in SLC25A12 was studied using Lewontin's standardised measure of disequilibrium $D^{\prime}$, calculated using the haploxt program. ${ }^{11}$ Haplotype analysis was carried out using the TDTPHASE v2.40 program, ${ }^{12}$ using expectation-maximization (EM) algorithm estimation of uncertain haplotype frequencies and the 'robust permutation' option.

Case-control association analysis was carried out using one proband selected at random from each multiplex family. Allele frequency differences between unrelated affected cases from the multiplex sample and controls were analysed using Fisher's exact test.

\section{Results and discussion}

Three SNPs previously shown to be associated with autism have been genotyped in 261 multiplex families from the IMGSAC collection: rs2056202 and rs2292813 in the SLC25A12 gene, and rs3749004 in CMYA3.

Multipoint nonparametric linkage analysis was carried out using the SNPs and flanking microsatellite markers. Although the SLC25A12 and CMYA3 genes are several megabases proximal to the maximum linkage peak obtained in the IMGSAC data set, ${ }^{3}$ there was some evidence of linkage to these SNPs, with a multipoint MLS of 1.12 at marker rs3749004 and an MLS of 0.94 at markers rs2056202 and rs2292813. The TDT results are presented in Table 2 . There is no evidence of association between autism and either the two SNPs within SLC25A12 or rs3749004 in CMYA3. Ramoz et $\mathrm{al}^{7}$ reported preferential transmission of the most frequent allele $(G)$ of both SNPs in SLC25A12. The same alleles are only very weakly overtransmitted in our data set. Similarly, only a modest overtransmission of the rs3749004 allele A was observed, mostly accounted for by maternal transmissions.

Haplotypes were constructed for the two SNPs within SLC25A12. The two SNPs were in strong $\operatorname{LD}\left(D^{\prime}=1\right.$, $\left.r^{2}=0.62\right)$, as reported in the previous study. ${ }^{7}$ All three observed haplotypes were evenly transmitted to affected offspring with no difference between maternal and paternal transmissions (Table 3 ).

The TDT design is dependent on transmissions from heterozygote parents to affected offspring, resulting in only a subgroup of families being informative for each marker. For this reason, we also tested the role of these SNPs using a more powerful case-control design. Genotype and allele frequencies were compared between unrelated cases from the multiplex families (one proband selected at random from each multiplex family) versus a group of 174 Caucasian controls (Table 4). Given the possibility of a sex-limited effect in autism, ${ }^{3}$ analysis was also carried out for male and female individuals separately (data not shown). No significant differences were detected, supporting the results obtained using the TDT design.

In summary, our study failed to replicate the associations reported by Ramoz et al ${ }^{7}$ and Faham et al, ${ }^{8}$ suggesting that the tested variants in SLC25A12 and CMYA3 are not likely to contribute strongly to autism susceptibility. The lack of association in our study possibly could be explained by the

Table 2 TDT analysis

\begin{tabular}{|c|c|c|c|c|c|c|c|c|c|c|}
\hline \multirow[b]{2}{*}{ Allele } & \multirow[b]{2}{*}{$\%^{\mathrm{a}}$} & \multicolumn{3}{|c|}{ Paternal } & \multicolumn{3}{|c|}{ Maternal } & \multicolumn{3}{|c|}{ Combined } \\
\hline & & $T R$ & $N T$ & $\chi^{2}(P)^{\mathrm{b}}$ & $T R$ & $N T$ & $\chi^{2}(P)^{\mathrm{b}}$ & $T R$ & $N T$ & $\chi^{2}(P)^{b}$ \\
\hline rs3749004.A & 88.5 & 42 & 34 & $0.84(0.32)$ & 41 & 26 & $3.36(0.11)$ & 91 & 68 & $3.36(0.11)$ \\
\hline rs2292813.G & 91.8 & 35 & 24 & $2.05(0.13)$ & 25 & 22 & $0.19(0.60)$ & 64 & 50 & $1.72(0.17)$ \\
\hline rs2056202.G & 87.3 & 39 & 25 & $3.06(0.12)$ & 42 & 46 & $0.18(0.70)$ & 90 & 80 & $0.59(0.45)$ \\
\hline
\end{tabular}

${ }^{a}$ Allele frequency in the parents.

${ }^{b}$ Empirical P-value calculated by ASPEX sib_tdt.

Table 3 TDT analysis of haplotypes rs2292813-rs2056202

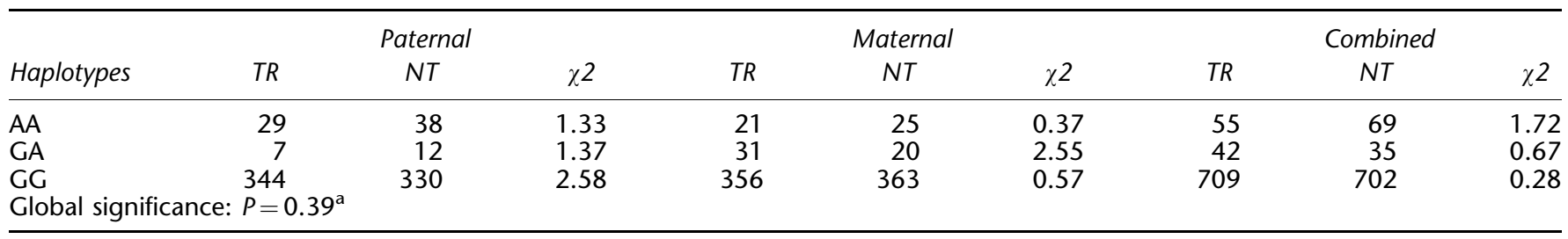

${ }^{\text {a }}$ Significance level calculated by TDTPHASE using 1000 permutations. 
Table 4 Distribution of genotype and allele frequencies

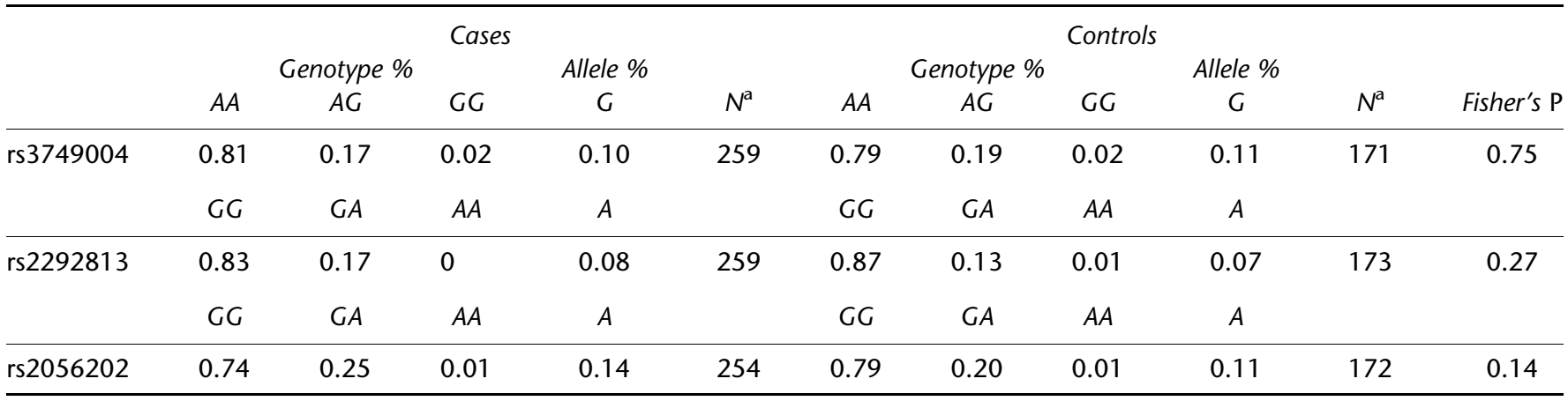

${ }^{a}$ Number of genotyped individuals.

presence of heterogeneity between the original and our data set. However, the populations used in the studies by Ramoz et $a l^{7}$ and Faham et $a l^{8}$ as well as the IMGSAC family collection, are of Caucasian origin. Therefore, it is unlikely that major differences in allele frequency or LD structure are present between the data sets. ${ }^{13}$

Ramoz et $\mathrm{al}^{7}$ estimated a genotype relative risk of 2.4-4.8 for individuals homozygous at the risk haplotype in SLC25A12, and the power of our sample size should be sufficient to detect such an effect. ${ }^{14}$ However, this may be an overestimation of the true effect size; therefore, failure to replicate the previous findings could also be due to insufficient power of our sample size to detect a minor gene effect. It is possible that these conflicting results may be resolved with further investigation using increased sample size and typing extra SNPs.

Although we could not confirm the evidence for an involvement of SLC25A12 and CMYA3 in autism susceptibility, the chromosome $2 \mathrm{q} 24-\mathrm{q} 33$ region remains an area of strong interest given the number of overlapping linkage findings. Analysis of additional positional candidate genes and high-resolution LD mapping studies are in progress and will hopefully lead to the identification of one or more functional gene variants involved in autism aetiology.

\section{Acknowledgements}

We thank all the families and professionals who have participated in the IMGSAC study. This study was funded in part by support from Telethon-Italy (GGP030227), Fondazione Cassa di Risparmio in Bologna, University of Bologna, the UK Medical Research Council, The Wellcome Trust, BIOMED 2 (CT-97-2759), EC Fifth Framework. AJB is the Cheryl and Reece Scott Professor of Psychiatry. APM is a Wellcome Trust Principal Research Fellow.

\section{References}

1 Pickles A, Bolton P, Macdonald $\mathrm{H}$ et al: Latent-class analysis of recurrence risk for complex phenotypes with selection and measurement error: a twin and family history study of autism. Am J Hum Genet 1995; 57: 717-726.

2 International Molecular Genetics Study of Autism Consortium: A genomewide screen for autism: strong evidence for linkage to chromosomes 2q, 7q, and 16p. Am J Hum Genet 2001; 69: $570-581$.

3 Lamb JA, Barnby G, Bonora E et al: Analysis of IMGSAC autism susceptibility loci: evidence for sex limited and parent of origin specific effects. J Med Genet 2005; 42: 132-137.

4 Philippe A, Martinez M, Bataille-Guillot $\mathrm{M}$ et al: Genome-wide scan for autism susceptibility genes. Hum Mol Genet 1999; 8 $805-812$.

5 Buxbaum JD, Silverman JM, Smith CJ et al: Evidence for a susceptibility gene for autism on chromosome 2 and for genetic heterogeneity. Am J Hum Genet 2001; 68: 1514-1520.

6 Shao Y, Raiford KL, Wolpert CM et al: Phenotypic homogeneity provides increased support for linkage on chromosome 2 in autistic disorder. Am J Hum Genet 2002; 70: 1058-1061.

7 Ramoz N, Reichert JG, Smith CJ et al: Linkage and association of the mitochondrial aspartate/glutamate carrier SLC25A12 gene with autism. Am J Psychiatry 2004; 161: 662-669.

8 Faham M, Zheng J, Moorhead M et al: Variation scanning on tag arrays (VSTA): a multiplexed assay used to scan 1,000 amplicons for variation in hundreds of samples. Proc Natl Acad Sci USA 2005, (in press).

9 Hennig BJ, Hellier S, Frodsham AJ et al: Association of low-density lipoprotein receptor polymorphisms and outcome of hepatitis $\mathrm{C}$ infection. Genes Immun 2002; 3: 359-367.

10 Hinds DA, Risch N: The ASPEX package: affected sib-pair exclusion mapping http://aspex.sourceforge.net/.

11 Abecasis GR, Cookson WO: GOLD - graphical overview of linkage disequilibrium. Bioinformatics 2000; 16: 182-183.

12 Dudbridge F: Pedigree disequilibrium tests for multilocus haplotypes. Genet Epidemiol 2003; 25: 115-221.

13 Rosenberg NA, Pritchard JK, Weber JL et al: Genetic structure of human populations. Science 2002; 298: 2381-2385.

14 Risch N, Merikangas K: The future of genetic studies of complex human diseases. Science 1996; 273: 1516-1517. 